\title{
Mathematical Modelling of Beauveria bassiana
}

\author{
Subhoshmita Mondal', Sarangam Majumdar ${ }^{2, *}$ \\ ${ }^{1}$ Department of Chemical Engineering, Jadavpur University, Kolkata-700032, India \\ ${ }^{2}$ Department of Mathematics, National Institute of Technology, Rourkela, Odisha -769008, India \\ *Corresponding Author: majumdarsarangam@yahoo.in
}

Copyright $(2014$ Horizon Research Publishing All rights reserved.

\begin{abstract}
The isolate of white muscardine, Beauveria bassiana was isolated from soil using SDAY media. The experiment was studied under submerged culture in selective broth at varied interval of days, in view of dry weight of hyphal biomass. In this presentation we want to introduce a mathematical growth model of Beauveria bassiana and the perspective of stochastic analysis of this fungus and compare with experimental data.
\end{abstract}

Keywords Beauveria bassiana, Mathematical Model, Hyphal Growth, Stochastic Analysis

\section{Introduction}

Biopesticides are certain types of pesticides derived from such natural materials as animals, plants, bacteria, and certain minerals. Microbial pesticides consist of a microorganism (e.g., a bacterium, fungus, virus or protozoan) as the active ingredient. Microbial pesticides can control many different kinds of pests, although each separate active ingredient is relatively specific for its target pests. For example, there are fungi that control certain weeds, and other fungi that kill specific insects [1]. Biopesticides often are effective in very small quantities and often decompose quickly, thereby resulting in lower exposures and largely avoiding the pollution problems caused by conventional pesticides. When used as a component of Integrated Pest Management (IPM) programs, biopesticides can greatly decrease the use of conventional pesticides, while crop yields remain high [2]. The paper outlines the current state of knowledge of insect fungal pathogens as it relates to their present use and future potential as mycoinsecticides.

Intensified use of chemical insecticides has resulted in the accumulation of toxic compounds potentially hazardous to humans and environment[3].Due to stringent environmental protection law, imposed by different statutory bodies of both central and state governments, application of chemical pesticides in agriculture is gradually been restricted. Processes like bioremediation and usage of naturally growing microorganisms as pesticides for protecting agricultural crops has a great impact inherently leads to green technology. Furthermore, their complex mode of action makes it unlikely that resistance could be developed to a bio-pesticide. Biopesticides offer a unique opportunity to developing countries to explore and develop their own natural biopesticide resources in crop protection. Such endeavours will aid in conserving cash reserves, improving safety to applicators and consumers and protecting the environment. However, care must be taken to ensure that any newly introduced natural product, be it a microbial agent or secondary metabolite, a plant extract or a living organism, poses no threat to the operator, the environment or the consumer before it is introduced into crop-protection systems. Although most biopesticides are much less hazardous to non-target species, the fact that they are natural does not automatically make them safe [4]. As of early 2013 there were approximately 400 registered biopesticide active ingredients and over 1250 actively registered biopesticide products. This paper preferably includes the entamopathogenic family of genera fungi, causing disease and act as a killer to generally the agricultural pests.

Entomopathogenic fungi were among the first organisms to be used for the biological control of pests. More than 700 species of fungi from around 90 genera are pathogenic to insects. Most are found within the deuteromycetes and entomophthorales. Some insect-pathogenic fungi have restricted host ranges, while other fungal species have a wide host range, with individual isolates being more specific, for example, Metarhiziumanisopliae and Beauveria bassiana [5]. Amongst them, Beauveria bassiana is the most favorable and eminent for its anti-pest activity, natural soil borne insect pathogens and are currently being reviewed as an effective alternative to chemical pesticides.

In 1912, Vuillemin first established genus Beauveria. About 14 species of Beauveria used as entomopathogenic fungi was listed in 1954, by Mac-Leodsuch as -Beauveria bassiana, Beauveriatenella, Beauveriadensa, Beauveria brongniartii etc. First experimental proof of parasitism of fungus Beauveria bassiana by Agostino Bansi was observed on silkworm (Bombyxmori)[6,7].Traditionally, due to the comparatively large heterogenicity differentiation of Beauveria spp. is done based on shape and size of the conidia.Because some researchers showed that spore shape could alter after culturing, [8] a number of molecular 
techniques were developed for identification of species. The infection process through insect cuticle is a asexual pathogenic life cycle. In presence of specific insect host, the conidiospores germinate on surface of cuticle and penetrates the insect integument directly with the development of hyphal tubes. When it is having penetrated cuticle, fungus alters its growth morphology and produce hyphal bodies, which circulates in the haemolymph and proliferate by budding. After insect death, fungal growth reverse back to typical hyphal form[9]. Ingression through the cuticle is facilitated by enzymatic degradation, producing chitinase, lipase and protease $[10,11]$. The fungi produce secondary metabolites including non-peptide pigments and polyketides (oosperin, bassianin and tenellin), nonribosomally synthesised peptides (beauvericin, bassianolides and beauveriolides) [12].

An interesting feature of Beauveria is the high host specificity of many isolates. In India it is most successfully utilized for controlling rice hispa in rice field and red spider Moth and Mosquito bug in Tea gardens. Tea is a perennial plantation crop grown under monoculture providing favorable conditions for a variety of pests. The concept of pest control has undergone a considerable change over the past few decades. The tea industry is one of the oldest organized industries in India and exported all over the world. Tea is grown in 13 states and Assam, West Bengal and Andhra Pradesh are the largest producers [13]. Total consumption of tea was estimated 4 million tonnes in 2010, with an increase of 5.6 percent. There is a steady increase in the production over the years since its day of first cultivation, which is due to extensive cultivation, improved technology, nutrition and fertility management [14], has introduced high yielding clones and longer pruning cycle. These factors, on the other hand, have encouraged biotic stresses like insect pests and diseases to limit the productivity of this crop [15]. More than one thousand species of arthropod pests are known to attack tea all over the world, though only about 300 species of insects are recorded from India in that 167 species from North-East India [16], resulting 11 to $55 \%$ annual loss in yield. For this reason, researchers have opted for biopesticides containing spores of entomopathogenic fungi. They are a novel strategy for controlling pests as well as are not harmful for the consumers. The present investigation is done from Northern and Southern parts of India having dense tea cultivation.

In the context of mathematical modeling of fungal development some authors are proposed several techniques. Park [17] introduced mathematical models for macroscopic development of fungal mycelia. Davidson et al.[18,19]studied large-scale behaviour of fungal mycelia ,travelling waves and pattern formation in a model for fungal development. In this paper we are studied mathematical and experimental growth model of Beauveria bassiana and some perspectives in this biological phenomenon. In the next couples of section we are organized as follows: in the next section material and methods are highlighted. In section 3 mathematical models is presented .Finally discussions of this approach are drawn in section 4 .

\section{Materials and Methods}

\subsection{Isolation and Identification}

Isolation was done from soils of four different tea plantation areas of north eastern and south eastern India. The samples were inoculated in SDAY (Sabouraund's dextrose agar, with $1 \%$ of yeast extract) media in petri plates at $\mathrm{pH} 5.6$, $25^{\circ} \mathrm{C}$ in total darkness for 5 days. After growth of inoculums, they were sub cultured on selective antibiotics like penicillin, gentamycin, streptomycin and chloramphenicol for single spore culture. The spore culture without any contamination is subjected to dye staining (Lactophenol cotton Blue) to confirm the fungal nature of the isolated culture, shown in figure 1. Field emission scanning electron microscopy was to identify the morphology, where conidial length and width were $2.27 \pm 0.22 \mu \mathrm{m}$ and $1.86 \pm 0.33 \mu \mathrm{m}$, respectively with length/width ratio of $1: 2$, shown in figure 2 . The conventional molecular sequencing characterized the genus and species of the fungal inoculums.

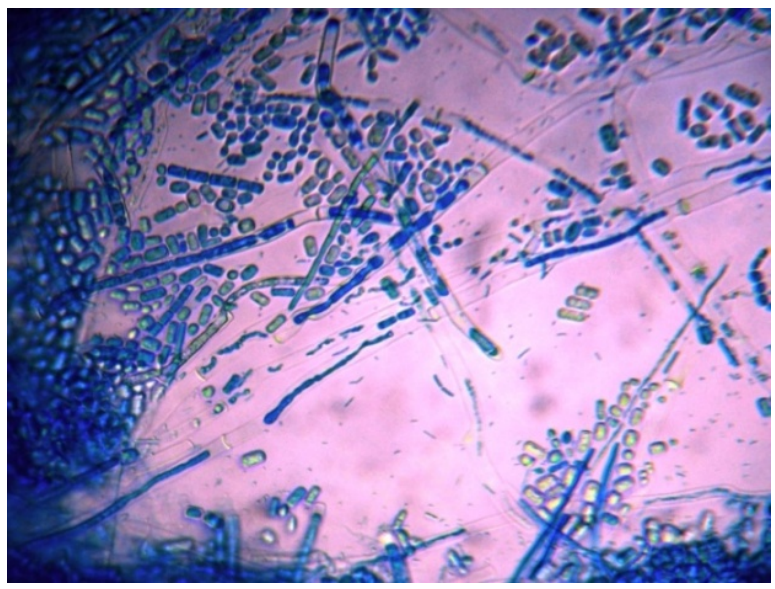

Figure 1. Lactophenol cotton blue staining of fungal inoculum seen under Phase contrast Microscope.

\subsection{Determination of Fungal Biomass}

Erlenmeyer flasks $(250 \mathrm{~mL})$ containing $100 \mathrm{~mL}$ of Sabouraund's dextrose broth were incubated with $1 \mathrm{~cm}^{2}$ discs of Sabouraund's dextrose (SDA) of actively growing mycelium of $B$. bassiana. The inoculated flasks were incubated at $25^{\circ} \mathrm{C}-30{ }^{\circ} \mathrm{C}$ for 5 days. $30 \mathrm{~mL}$ broth containing mycelium at interval of two days was collected and centrifuged (REMI centrifuge) at $15000 \mathrm{rpm}, 4^{\circ} \mathrm{C}$ for 15 mins. The supernatant was discarded and separated biomass was washed with phosphate buffer ( $\mathrm{pH} 7)$ solution. It was then transferred into a pre-weighed aluminum cup individually dried at $60^{\circ} \mathrm{C}$ for 12 hours. The exact weight of fungal bio mass is determined by subtracting the weight of dry cup from that of the cup containing dried biomass. The 
experiment determines the hyphal growth of B.bassiana at interval of days.
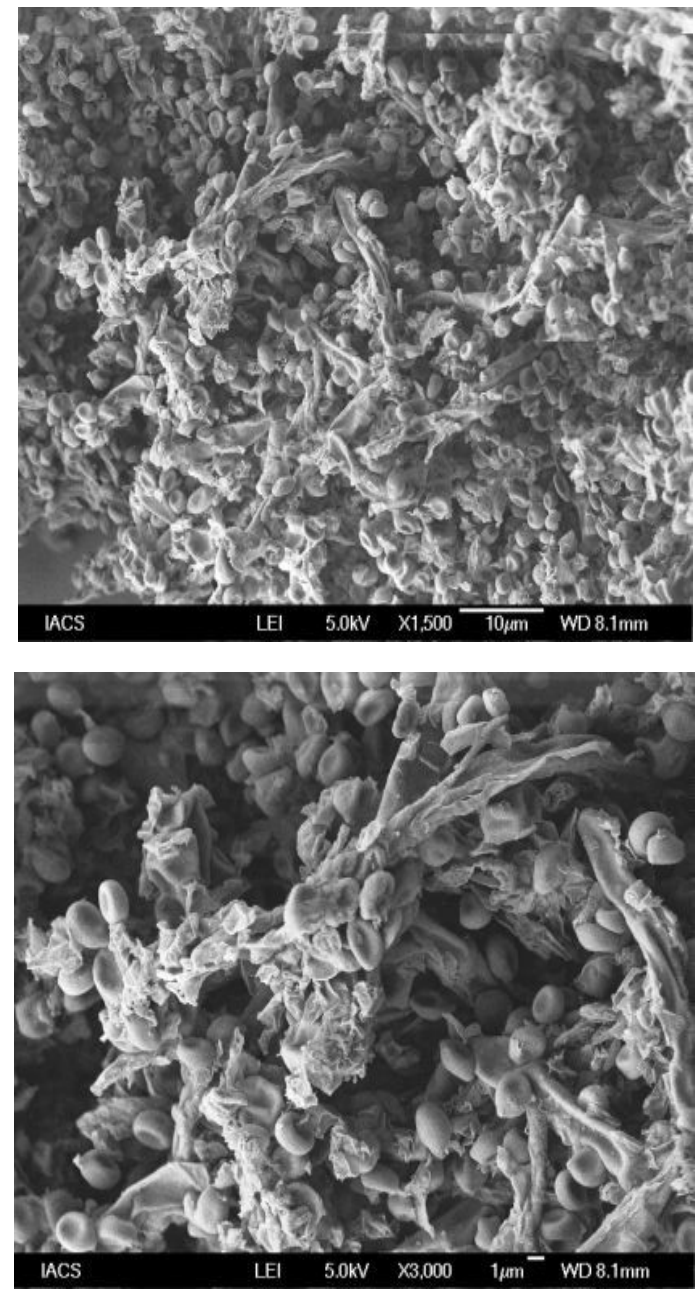

Figure 2. FE-SEM of profuse fungal inoculum (Beanveria bassiana) at different magnifications

\subsection{Glucose estimation}

The supernatant is further used glucose content after the growth of fungal mycelium at interval of days. Glucose is estimated by 3,5-dinitrosalicylic acid (DNS) assay method considered as the carbon source in SDAY medium for fungal growth, shows total reducing sugar. Briefly, 1gm DNS reagent, $20 \mathrm{mg}$ crystallized phenol dissolved in $50 \mathrm{mg}$ $\mathrm{Na}_{2} \mathrm{SO}_{3}$ with $1 \% \mathrm{NaOH} 2(\mathrm{~N})$ in a $100 \mathrm{~mL}$ volumetric flask, along with $40 \%$ solution of sodium potassium tartarate (Rochelle's salt). Standard curve of glucose solution is prepared using different concentrations of glucose and glucose-reagent mixture $(1: 1)$ are heated at $100^{\circ} \mathrm{C}$ for 5 minutes followed by addition of $1 \mathrm{~mL}$ Rochelle's salt analyzed in Spectophotometreat $510 \mathrm{~nm}$ with respect to blank sample.

Supernatants of harvested broth of 2 days interval of individual culture are taken for glucose estimation for approximately identifying substrate glucose utilization by $B$. bassiana. $30 \mathrm{~mL}$ of broth are centrifuged (REMI centrifuge) at $15,000 \mathrm{rpm}, 4^{0} \mathrm{C}$ for 15 minutes and appropriately diluted supernatant of harvested broth are used for glucose content in the isolated fungal culture under same assay conditions.

\section{Mathematical Model}

Now we develop a mathematical approach of this fungal growth. Based on this experiment we study this biological phenomena using reaction diffusion equation. Let us consider diffusion in three dimensions. Assume $S$ be an arbitrary surface enclosing a volume $V$ of Beauveria bassiana. Then the general conservation equation is $\frac{\partial}{\partial t} \int_{V} A(\mathbf{x}, t) d v=-\int_{S} \mathbf{J} \cdot \mathbf{d} \mathbf{s}+\int_{V} f . d v \quad(1)$. Where $A$ is the concentration of the nutrient (broth), $\mathbf{J}$ is flux of nutrient and $\mathrm{f}$ is the source of nutrient which is a function of $A, \mathbf{x}$ and $\mathrm{t}$. Now applying divergence theorem to the surface integral and assume that $A(\mathbf{x}, \mathrm{t})$ is continuous, then from equation (1) we get $\int_{V}\left[\frac{\partial A}{\partial t}+\nabla . \mathbf{J}-f(A, \mathbf{x}, t)\right] d v=0 \quad$ (2). Since the volume $\mathrm{V}$ is arbitrary the integrant must be zero. Therefore we arrive at the equation $\left[\frac{\partial A}{\partial t}+\nabla . \mathbf{J}-f(A, \mathbf{x}, t)\right]=0$ From the classical diffusion (Fickian diffusion) we known $\mathbf{J}$ $=-D \nabla A$ (in one dimension) and (3) becomes $\frac{\partial A}{\partial t}=$ $\nabla .(D \nabla A)+f(A, \mathbf{x}, t)(4)$, where $\mathrm{D}=\lim _{\substack{\Delta x \rightarrow 0 \\ \Delta t \rightarrow 0}} \frac{(\Delta x)^{2}}{2 \Delta t}$ is known as diffusivity and the particle moves randomly along a line in fixed steps $\Delta x$ that are taken in a fixed time $\Delta t$ [20].We assume a logistic growth asf $=\operatorname{rA}\left(1-\frac{A}{K}\right)$, where $\mathrm{r}>0$ is known as growth rate and $\mathrm{K}$ is the carrying capacity [21]. Then equation (4) becomes $\frac{\partial A}{\partial t}=\nabla \cdot(D \nabla A)+\mathrm{rA}\left(1-\frac{A}{K}\right)$ (5).

\section{Discussions}

Mathematical model and the analysis give several interpretations about the pattern formation, sigmoidal growth of the fungi .This growth is depended on nutrient availability. Figure 3 shows the growth of the Beauveria bassiana .

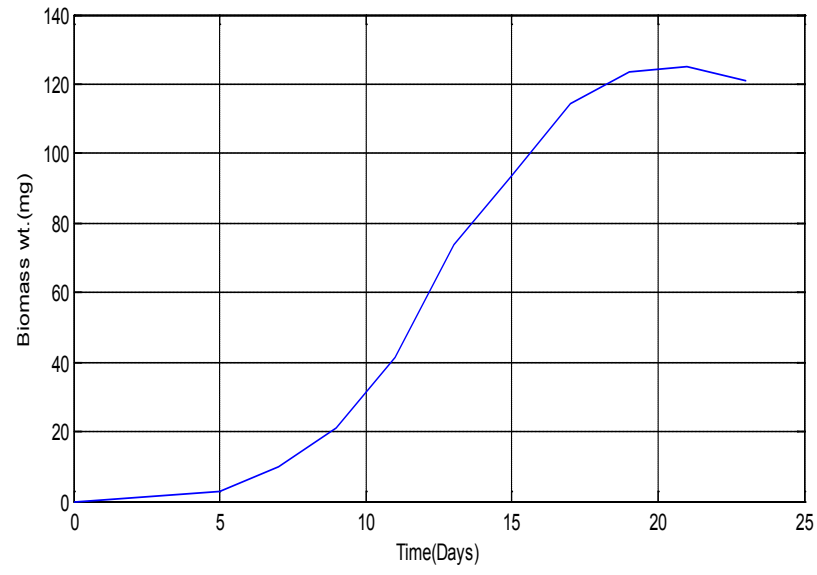

Figure 3. Growth of Beauveria bassiana 
Mathematical modeling approach is the ideal way to understand bio-mechanism of hyphal mass growth and thigmotropism. This quantitative analysis is efficient for studying molecular genetics and the role in the host environment. We can also use the convection diffusion reaction equation and Focker- Planck equation to understand deeply this biological phenomenon and the hyphal network in future.

\section{Acknowledgements}

Department of Chemical Engineering (Jadavpur University, Kolkata, India) is gratefully acknowledged for providing necessary facilities in laboratory for microbial work. We thank Department of Plant pathology (ITCC, ICAR unit, New Delhi) for molecular identification of fungal inoculums.

\section{REFERENCES}

[1] Coombs, Amy. "Fighting Microbes with Microbes". The Scientist. Retrieved 18 April 2013.

[2] Environmental Protection Agency of the USA (2012) Regulating Biopesticides. Accessed on 20 April 2012.

[3] R. J. Cook, K. F. Baker: The Nature and Practice of Biological Control of Plant Pathogens. Amer. Phytopathol. Soc., Minnesota (1983) p. 539.

[4] Menn JJ and Hall FR, Biopesticides Present Status and Future Prospects, in Biopesticides Use and Delivery, ed by Hall FR and Menn JJ, Humana Press, Totowa, New Jersey, pp. 1-10 (1999).

[5] Florez, F. J. P. (2002). Fungi for coffee berry borer controlColombia. 35th annual meeting of the Society of Invertebrate Pathology, Foz Do Iguassu, Brazil.

[6] Brady B.L.K. 1979a. Beauveria bassiana .CMI descriptions of pathogenic fungi, No. 602.p.1964-1995. Commonwealth Mycological Institute, Kew, Surrey, England.

[7] Ainsworth GC (1956). Agostino Bassi, 1773-1856. Nature 177: 255-257.

http://dx.doi.org/10.1038/177255a0. Bassi A (1836). Del mal del segno e di altre malattie dei bachi da seta. Parte seconda. Practica. Tipografia Orcesi, Lodi : 58.

[8] Townsend R.J., Glare T.R., Willoughby B.E. 1995. The fungi Beauveria spp. causes grass grub population collapse in some Waikato pastures. Proc. of the 48th New Zealand Plant Protection Conference 48: 237-241.
[9] St Leger, R. J., T. M. Butt, et al. (1989a). "Production in vitro of appressoria by the entomopathogenic fungus Metarhiziumanisopliae.” Exp. Mycol. 13: 274-288

[10] Zacharuk R.Y. 1970. Fine structure of the fungus Metarhiziumanisopliae infecting three species of larval Elateridae. III. Penetration of the host integument. J. Invertebr. Pathol. 15:372-396.

[11] Hassan A.E.M., Charnley A.K. 1989. Ultra structural study of the penetration by Metarhiziumanisopliae through Dimilin -affected cuticle of Manducasexta. J. Invertebr. Pathol. 54:117-124.

[12] Elsworth, J. F. and J. F. Grove (1977). "Cyclodepsipeptides from Beauveria bassiana Bals. Part1. Beauverolides $\mathrm{H}$ and I."J ChemSoc [Perkin 1] 3: 270-3.

[13] Muraleedharan, N.: Tea research in India. In: Plantation crops research : An overview (Eds.: J. Thomas, T.K. Hrideek, Joseph Thomas and K.M.Kuruvilla). PLACROSYM XVII, Indian Cardamom Research Institute, Spices Board India, Idukki, Kerala. pp. 81-88 (2006).

[14] Saraswathy, R., S. Suganya and P. Singaram: Environmental impact of nitrogen fertilization in tea eco-system. J. Environ. Biol., 28, 779-788 (2007)

[15] Gurusubramanian, G.: Safe and economic pest management in tea, shade trees and green crops. In: Manual on tea culture for condensed course (Eds.: A.K. Dutta, N. Ahmed, B. Kotoky and D. Burugohain). Tocklai Experimental Station, TRA, Jorhat Assam Printing Works Private Limited, Jorhat, Assam, India. pp. 113-127 (2005)

[16] Das, G.M.: Pests of tea in North East India and their control. Memorandom No. 27, Tocklai Experimental Station, Tea Research Association, Jorhat, Assam, India .pp. 169-173 (1965).

[17] A.W. Park, Mathematical models for macroscopic development of fungal mycelia, M.Sc. Dissertation, Dept. of Mathematics \& Computer Science, University of Dundee, (1996).

[18] F.A. Davidson, B.D. Sleeman, A.D.M. Rayner, J.W. Crawford and K. Ritz, Large-scale behaviour of fungal mycelia, Mathl. Comput.Modelling24 (lo), 81-87, (1996).

[19] F.A. Davidson, B.D. Sleeman, A.D.M. Rayner, J.W. Crawford and K. Ritz, Travelling waves and pattern formation in a model for fungal development, J. Math.Biol. $35,589-608,(1997)$

[20] J.D.Murray, Mathematical Biology: I. An Introduction, Third Edition, Springer (2002).

[21] S.H. Strogatz. Nonlinear Dynamics and Chaos: with Applications in Physics, Biology, Chemistry, and Engineering. Addison-Wesley Publishing, Reading, MA, 1994. 